\title{
Introduction to this special issue on tumor targeting
}

\author{
David M. Goldenberg
}

Published online: 11 April 2012

(C) International Society of Oncology and BioMarkers (ISOBM) 2012

This issue of Tumor Biology is devoted to tumor targeting with radioconjugates, and includes 12 diverse articles, with another published in a prior issue [1]. It was not a challenge for me to assemble these papers because the authors were participating in three conferences held on this subject during the past year in Uppsala, Stockholm, and Washington, D.C. Having organized 13 meetings devoted to radioimmunoconjugates for cancer imaging and therapy since 1979, I am impressed with the enthusiasm and progress being made despite the long history of this field. Indeed, there have been important clinical advances, both in antibody-based radiotherapeuticals (e.g., tositumomab and ibritumomab tiuxetan) as well as radiolabeled peptides (e.g., somatostatin analogs), with a considerable number of agents now in various stages of clinical development. Robert Sharkey and I summarized progress in cancer radioimmunotherapy recently [2], so I will not expand on this here.

The articles in this issue reflect the broad advances being made in better and simpler labeling of antibodies and peptides with radionuclides, including positron- and alpha emitters, new antibody and non-antibody constructs, and also different delivery strategies, such as pretargeting, as well as a better understanding of basic problems and prospects. ${ }^{18} \mathrm{~F}$ fluorodeoxyglucose has been the mainstay in cancer imaging in particular, and nuclear medicine in general, over the past decade or more. We are now witnessing the improvement and application of ${ }^{18} \mathrm{~F}$ chemistry to other molecules, as well as ${ }^{68} \mathrm{Ga}$ constructs, thus complementing the advances in ${ }^{99 \mathrm{~m}} \mathrm{Tc}$ chemistry made in the prior decade. Whereas ${ }^{131} \mathrm{I}$, ${ }^{90} \mathrm{Y}$, and ${ }^{177} \mathrm{Lu}$ have been the most popular radionuclides for

D. M. Goldenberg $(\bowtie)$

Garden State Cancer Center,

Center for Molecular Medicine and Immunology,

Morris Plains, NJ, USA

e-mail: dmg.gscancer@att.net therapy, the emergence of alpha emitters from the laboratory to the clinic is now a reality. Different antibody forms, as well as non-antibody targeting agents (e.g., affibody), also are being studied as alternatives to $\operatorname{IgG}$ forms, particularly for imaging. Finally, separation of the targeting moiety from the radionuclide effector, as with pretargeting, is now in clinical trials for cancer therapy, and soon for molecular imaging.

Part of the satisfaction in assembling these articles is the interaction with a group of devoted and enthusiastic scientists from various countries, to whom I am grateful for their participation and submission of their manuscripts to peer review and revision. I am most indebted to the editor-in-chief of Tumor Biology, Torgny Stigbrand, who accepted the major role and chore of conducting the reviews and interactions with the authors, and who conceived of this special issue. My association with this journal goes back to its formation over 30 years ago, when I enjoyed a relationship to its former editors, William H. Fishman (1980-1983), Hidematsu Hirai (19801983), A. Munro Neville (1984-1994), and Sabine von Kleist (1995-1999), as well as many editorial board members who helped establish the field of cancer biomarkers as part of the general topic of oncodevelopmental biology and medicine, such as the pioneers of AFP, CEA, PSA, etc. These were also the true pioneers of personalized medicine approaches, always relating serum marker results to the clinical course and assessment of therapeutic outcome.

New methods of cancer targeting leading to more specific and individualized therapies is an important part of the excitement in cancer research in this new century, and targeted radionuclides is just one such form in addition to drugs, toxins, cytokines, and other therapeutic modalities that can benefit from improved localization, thus hopefully increasing the therapeutic index. With the plethora of journals that include such topics, especially as online journals expand rapidly, it is a convenient and stimulating effort to 
offer an issue focused on a single aspect of the broad field of tumor targeting. I trust that the readership and authors will be pleased with the outcome.

Conflicts of interest The author is an officer of, and has a financial interest in, Immunomedics, Inc.

\section{References}

1. Laverman P, D’Souza CA, Eek A, McBride WJ, Sharkey RM, Oyen WJ, Goldenberg DM, Boerman OC. Optimized labeling of NOTAconjugated octreotide with F-18. Tumor Biol. 2012;33(2):427-34. Epub 2011 Oct 19.

2. Sharkey RM, Goldenberg DM. Cancer radioimmunotherapy. Immunotherapy. 2011;3(3):349-70. 